\title{
Charges and Electromagnetic Radiation as Topological Excitations
}

\author{
Manfried Faber \\ Atominstitut, Technische Universität Wien, Österreich, Austria \\ Correspondence should be addressed to Manfried Faber; faber@kph.tuwien.ac.at
}

Received 11 May 2017; Accepted 4 July 2017; Published 22 August 2017

Academic Editor: Ralf Hofmann

Copyright (C) 2017 Manfried Faber. This is an open access article distributed under the Creative Commons Attribution License, which permits unrestricted use, distribution, and reproduction in any medium, provided the original work is properly cited. The publication of this article was funded by $\mathrm{SCOAP}^{3}$.

\begin{abstract}
We discuss a model with stable topological solitons in Minkowski space with only three degrees of freedom, the rotational angles of a spatial Dreibein. This model has four types of solitons differing in two topological quantum numbers which we identify with electric charge and spin. The vacuum has a two-dimensional degeneracy leading to two types of massless excitations, characterised by a topological quantum number which could have a physical equivalent in the photon number.
\end{abstract}

\section{Introduction}

In our mathematical description of nature we use two different concepts. Following Einstein we formulate gravity in a geometrical language, whereas particle physics uses the algebraic formulae of quantum field theory. To unify the two very successful theories most physicists try quantized gravity. I go here into the opposite direction and follow first steps in a geometrical formulation of particle physics. We should find out from experiments the direction which we should take in our investigations. Nature may give us some hints about the mechanisms. I get my first intuition from the sine-Gordon model and its experimental realisation with a pendulum model. The mathematics of the sine-Gordon model is nicely described in [1]. Here I want only to repeat the most interesting physical pictures of this model. It is a fully relativistic model in $1+1 \mathrm{D}$, where the velocity of light corresponds to the propagation velocity $c$ of small amplitude waves. In the experimental realisation (see [1]), this velocity is of the order of $1 \mathrm{~m} / \mathrm{s}$. Besides waves, we find two types of particlelike excitations, kinks, and antikinks. They behave in many ways like particles. Their energy density is concentrated in a certain region in space with a total energy defining the mass. The three contributions to the energy, stress energy, potential energy, and kinetic energy have different dependencies on the velocity $v$ of a moving kink. The potential energy is decreasing; stress energy and kinetic energy are increasing with $v$.
As expected for a relativistic model, the three contributions nicely sum up to a mass increasing with $\gamma=1 / \sqrt{1-(v / c)^{2}}$. A moving kink is Lorentz contracted. In the mechanical model, we can easily imagine what nature does to decrease the size of the kink. To accelerate the pendula at the front of the kink the angle between them has to increase, leading to a smaller size of the kink. The pendulum model gives me some idea on how nature could work to realise the phenomena of special relativity. Even more impressive is the existence of two types of kinks, kinks and antikinks and their interaction. They behave like charged particles: kinks and kinks repel; kinks and antikinks attract each other. In soft collisions kinks behave similarly to billiard balls. In hard collisions the diameters of kinks shrink proportional to $1 / \gamma$ and get point-like. Further, we can observe how kinks and antikinks annihilate. In the mechanical model the annihilation due to friction effects gives rise to the emission of waves. In the abstract theoretical model solitons and antisolitons get through each other with a small time delay. Mathematically we can separate the various kink configurations in homotopy classes differing in their winding number. This is condensed in the relation $\Pi_{1}\left(\mathbb{S}^{1}\right)=\mathbb{Z}$ of homotopy theory.

I get a second hint from a simple model teaching us about the nature of $4 \pi$-rotations. I saw it for the first time in Figure 41.6 on page 1149 of "Gravitation" by Misner et al. [2]. A ball is attached with several wires to the surrounding, for example, 
with eight wires to the corners of a cube. Rotating the ball one or two times around some axis leads to a complete mess of the strings. But after a $4 \pi$-rotation one can disentangle the wires without moving the ball. We can learn from this model that a body which is connected to the surrounding returns only after a $4 \pi$-rotation to his original state. For a disconnected body this happens already after a $2 \pi$-rotation. This is mathematically formulated in the relation $\Pi_{1}\left(\mathbb{S}^{3}\right)=1$. There is a continuous transition between $4 \pi$-rotations and no rotation. This ball model gives me a hint on how possibly nature realises particles with spin $1 / 2$ just by connecting them with the surrounding.

Thirdly, I want to mention that, observing phenomena at and below the atomic scale, we always observe particles or clicks and never waves. Remember the double-slit experiment. In this interference experiment of electrons or photons, the wave-picture appears only after several hundreds or thousands of particles have been registered on the screen.

In the main part of this article, I describe a Lorentz covariant model which has stable topological excitations with properties of particles. In a certain sense, it is a generalisation of the sine-Gordon model to $3+1 \mathrm{D}$. Several features of this model were already described in a few articles [3-8]. In this article, I will mainly concentrate on topological questions.

\section{Definition of the Model}

We are using a scalar SO(3)-field in 3 + 1D Minkowski space. The only degrees of freedom of this model are therefore three rotational angles, for example, the three Euler angles, describing the rotations of a spatial Dreibein. Work with $\mathrm{SU}(2)$ is simpler than using $\mathrm{SO}(3)$-matrices, that is, with $2 \times 2$-matrices. Since $\mathrm{SU}(2)$ is the double covering group of $\mathrm{SO}(3)$ there is an essential difference between $\mathrm{SU}(2)$ - and $\mathrm{SO}(3)$-fields. Every field configuration of an $\mathrm{SO}(3)$-field is twice realised by $\mathrm{SU}(2)$-fields. The two realisations differ by a transformation with the nontrivial center element, by a $2 \pi$ rotation. We have to remember this property using $\mathrm{SU}(2)$ matrices:

$$
\mathrm{Q}(x)=\mathrm{e}^{-\mathrm{i} \alpha(x) \vec{\sigma} \vec{n}(x)}=\cos \alpha(x)-\mathrm{i} \vec{\sigma} \vec{n}(x) \sin \alpha(x),
$$

at every site $x$ in $M_{4}$. The symbol $Q$ we are using reminds us of quaternions, invented by Rodrigues [9] in the year 1840 , to describe active rotations with the three imaginary quaternionic units $\mathbf{i}, \mathbf{j}, \mathbf{k}$. In (1), they are represented by Pauli matrices $\mathbf{i}:=-\mathrm{i} \sigma_{1}, \mathbf{j}:=-\mathrm{i} \sigma_{2}, \mathbf{k}:=-\mathrm{i} \sigma_{3} \cdot \vec{n}$ is a three-component unit vector and $\vec{\sigma} \vec{n}:=\sum_{i=1}^{3} \sigma_{i} n_{i}$ is the component of the Pauli matrices in direction of $\vec{n}$. Rotations are unit quaternions $Q=q_{0}-i \vec{\sigma} \vec{q}$ with $q_{0}^{2}+\vec{q}^{2}=1$. Their manifold is isomorphic to $\mathbb{S}^{3}$.

The idea for the definition of the dynamical part of the Lagrangian is its identification with the square of the area density on $\mathbb{S}^{3}$ in appropriate units of an action density. We start defining tangential vectors:

$$
\partial_{\mu} Q:=-\vec{i} \vec{\sigma} \vec{\Gamma}_{\mu} Q \quad \text { with } \vec{\sigma} \vec{\Gamma}_{\mu}:=\sum_{i=1}^{3} \sigma_{i} \Gamma_{\mu i},
$$

(tangential one-forms) to $\mathbb{S}^{3}$. We would like to emphasise that $\vec{A}_{\mu}=2 \vec{\Gamma}_{\mu}$ is a trivial connection but $\vec{\Gamma}_{\mu}$ is not. With the cross-product $\vec{R}_{\mu \nu}:=\vec{\Gamma}_{\mu} \times \vec{\Gamma}_{\nu}$ we can get the square of the area density $\vec{R}_{\mu \nu} \vec{R}^{\mu \nu}$ and define the Lagrangian in appropriate SI-units with $\alpha_{f}:=e_{0}^{2} / 4 \pi \epsilon_{0} \hbar c$ :

$$
\begin{aligned}
& \mathscr{L}:=\mathscr{L}_{\mathrm{dyn}}-\mathscr{H}_{\mathrm{pot}}:=-\frac{\alpha_{f} \hbar c}{4 \pi}\left(\frac{1}{4} \vec{R}_{\mu \nu} \vec{R}^{\mu \nu}+\Lambda\left(q_{0}\right)\right) \\
& \vec{R}_{\mu \nu}:=\vec{\Gamma}_{\mu} \times \vec{\Gamma}_{\nu}, \\
& \vec{\Gamma}_{\mu} \stackrel{(2)}{=} \frac{\mathrm{i}}{2} \mathrm{Sp}\left(\vec{\sigma} \partial_{\mu} Q Q^{\dagger}\right) .
\end{aligned}
$$

Up to a proportionality factor, the kinetic term $\mathscr{L}_{\text {dyn }}$ of this model agrees with the Skyrme term in the Skyrme model [10]. To get stable solitons, Skyrme suggested to use a mass term for the vector field $\vec{\Gamma}_{\mu}$ leading to Skyrmions with short-range forces which are accepted as approximations for nucleons. We want to describe particles with long-range Coulombic forces and have therefore to avoid the Skyrme term. The HobartDerrick theorem $[11,12]$ allows as additional terms only terms without derivative, a potential term, which we chose as

$$
\Lambda\left(q_{0}\right)=q_{0}^{2 m}
$$

Therefore, we have a two-dimensional manifold of degenerate vacua; the equatorial sphere $\mathbb{S}_{\text {equ }}^{2}$ is defined by $q_{0}=0$. The choice of the potential term (4) has two immediate physical consequences. There are two Goldstone bosons which we can identify with the two photon degrees of freedom. Further, we get nontrivial field configurations of finite energy which can be classified by $\Pi_{2}\left(\mathbb{S}_{\text {equ }}^{2}\right)=\mathbb{Z}$, by the map of $\mathbb{S}_{\text {equ }}^{2}$ to the sphere $\mathbb{S}_{\infty}^{2}$ at spatial infinity.

\section{Stable Solitons of Finite Energy}

Inserting the time-independent hedgehog ansatz

$$
\begin{aligned}
q_{0} & =\cos \alpha(r), \\
\vec{q} & =\vec{n}(\mathbf{r}) \sin \alpha(r), \\
\vec{n}(\mathbf{r}) & =\frac{\vec{r}}{r}, \\
\alpha(r) & \in\left[0, \frac{\pi}{2}\right]
\end{aligned}
$$

into Lagrangian (3), we get the Euler-Lagrange equation:

$$
\begin{array}{r}
\partial_{\rho}^{2} \cos \alpha+\frac{\left(1-\cos ^{2} \alpha\right) \cos \alpha}{\rho^{2}}-m \rho^{2} \cos ^{2 m-1} \alpha=0 \\
\text { with } \rho=\frac{r}{r_{0}}
\end{array}
$$




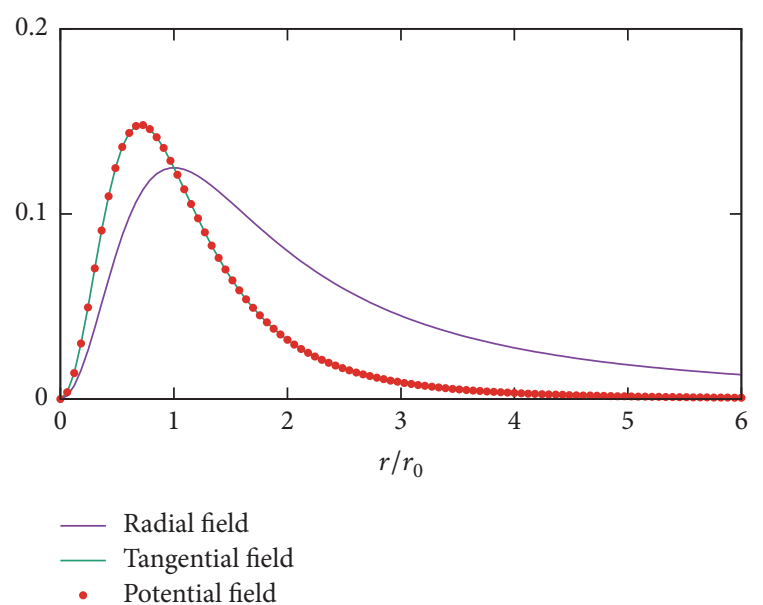

FIGURE 1: Contributions to the radial energy density according to (8) in units of $\alpha_{f} \hbar c / r_{0}$.

a nonlinear differential equation, which we can most easily solve for $m=3$. It has the simple solution

$$
\begin{aligned}
\alpha(r) & =\arctan \rho, \\
\sin \alpha(r) & =\frac{\rho}{\sqrt{1+\rho^{2}}}, \\
\cos \alpha(r) & =\frac{1}{\sqrt{1+\rho^{2}}} .
\end{aligned}
$$

leading to the radial energy density:

$$
h=\frac{\alpha_{f} \hbar c}{r_{0}}\left[\frac{\rho^{2}}{2\left(1+\rho^{2}\right)^{2}}+\frac{\rho^{2}}{\left(1+\rho^{2}\right)^{3}}+\frac{\rho^{2}}{\left(1+\rho^{2}\right)^{3}}\right]
$$

The radial dependencies of its three contributions are shown in Figure 1. At large distances the radial energy density agrees with the Coulombic energy density of a point charge. As one can clearly see, the singularity at the origin that pointcharges usually are suffering from is removed. The tangential and the potential energy density have equal shapes and decay faster. They lead to a deviation from the Coulomb interaction at distances in the order of $r_{0}$ and smaller. The total energy sums up to $E=\left(\alpha_{f} \hbar c / r_{0}\right)(\pi / 4)$. Comparing this result with the rest energy of an electron we get a value for the scale $r_{0}$ of $2.21 \mathrm{fm}$.

The hedgehog configuration, defined in (5), is schematically depicted in the upper diagram of Figure 2. It maps $\mathbb{R}^{3}$ to half of $\mathbb{S}^{3}$. This half-sphere is indicated in the lower diagram of Figure 2. By the electric field, the hedgehog is wired to the surrounding. If the center of the hedgehog is rotated by $4 \pi$, the tangled lines of constant $\vec{n}$-field can be disentangled without further rotation of the center, and the original configuration can get restored. This behaviour reminds us of the rotational property of spin-1/2 particles.

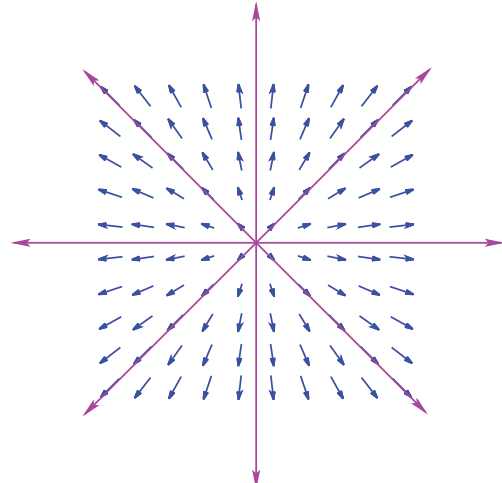

(a)

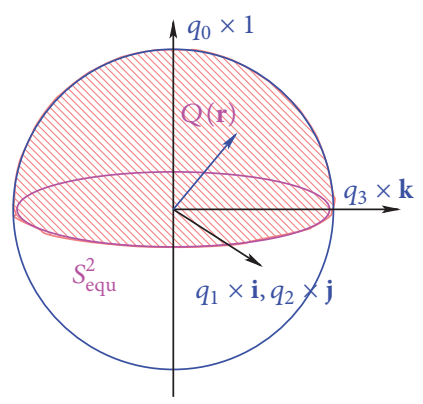

(b)

Figure 2: (a) Scheme of the hedgehog configuration (5). The small blue arrows show the imaginary part $\vec{q}=\vec{n}(\mathbf{r}) \sin \alpha(r)$ of the Qfield in an arbitrary plane through the origin. The long magenta lines indicate the electric field lines of a point charge, the lines of constant $\vec{n}$-field. (b) Half-sphere covered by the hedgehog configuration.

\section{Topological Quantum Numbers}

We can find a further relation to spin in the number of coverings of $\mathbb{S}^{3}$, the topological charge $\mathbb{Q}$ which we define in spherical coordinates $r, \vartheta, \varphi$ by

$$
\mathcal{Q}:=\frac{1}{2 \pi^{2}} \int_{0}^{\infty} \mathrm{d} r \int_{0}^{\pi} \mathrm{d} \vartheta \int_{0}^{2 \pi} \mathrm{d} \varphi \vec{\Gamma}_{r}\left(\vec{\Gamma}_{\vartheta} \times \vec{\Gamma}_{\varphi}\right)
$$

The configuration of (5) and Figure 2 results in $Q=$ $1 / 2$. Continuous modifications of the soliton field do not change $Q$ and the homotopy class of the configuration. The homotopy class can be changed by parity transformations $\Pi_{n}: \vec{n} \mapsto-\vec{n}$ in the internal space and by transformations with the nontrivial center element $z=-1$. The four types of solitons which we get by these transformations are shown in Table 1. Q-values with $q_{0} \geq 0$ are indicated by full blue arrows and with $q_{0} \leq 0$ by dashed green arrows. Besides the topological charge, the four configurations differ in the direction of the rotational axis of the Q-field at infinity which we will relate in (16)-(18) with the electric charge $Q_{\mathrm{el}}$. This field at large distances from the center determines the interaction with other solitons, attraction, or repulsion. The pairs of configurations with the same electric charge differ in the value $\pm 1 / 2$ of the topological charge, in the chirality. We can combine pairs of solitons with $Q= \pm 1 / 2$ either to 
TABLE 1: Soliton types differ by transformations with $\Pi_{n}$ and $z$. The signs of $\vec{n}$ and $q_{0}$ and the topological quantum numbers $Q_{\mathrm{el}}$ and $Q_{Q}$ are quoted. The configurations are spherically symmetric.

\begin{tabular}{|c|c|c|c|c|}
\hline Transf. & 1 & $z$ & $\Pi_{n}$ & $z \Pi_{n}$ \\
\hline$\vec{n}$ & $\vec{r} / r$ & $-\vec{r} / r$ & $-\vec{r} / r$ & $\vec{r} / r$ \\
\hline$q_{0}$ & $\geq 0$ & $\leq 0$ & $\geq 0$ & $\leq 0$ \\
\hline$Q_{\mathrm{el}}$ & -1 & 1 & 1 & -1 \\
\hline Q & $1 / 2$ & $1 / 2$ & $-1 / 2$ & $-1 / 2$ \\
\hline Diagram & 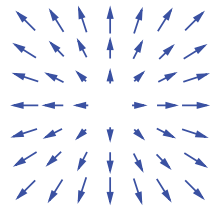 & 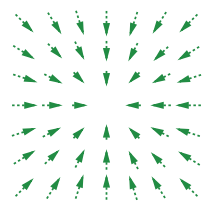 & 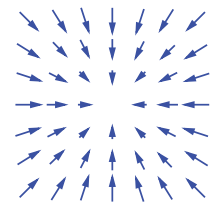 & 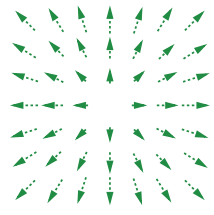 \\
\hline
\end{tabular}

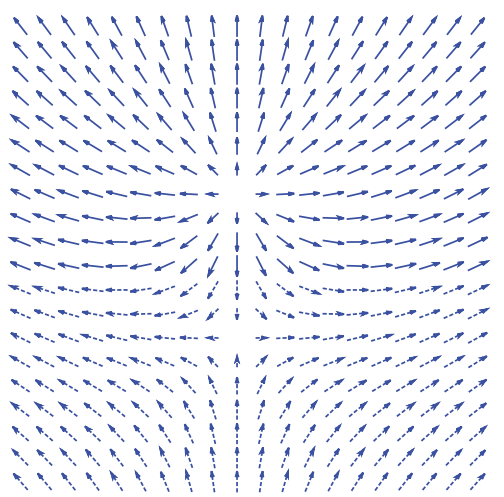

Figure 3: Dipole configuration with $S=1$. The small blue arrows show the imaginary part $\vec{q}=\vec{n}(\mathbf{r}) \sin \alpha(r)$ of the $Q$-field in an arbitrary plane through the centers of both solitons. Full arrows correspond to $q_{0} \leq 0$ and dashed arrows to $q_{0} \leq 0$.

$\mathbb{Q}=0$ or to $Q= \pm 1$. Since $S U(2)$ is the spin group and due to the above described properties of the soliton configurations under $4 \pi$ rotations we dare to identify the absolute value of the topological charge with the spin quantum number:

$$
s=|Q| \text {. }
$$

We would like to remember that, within $\mathrm{SO}(3)$, the two configurations which differ in the sign of $Q$ are identical.

Traversing the center of a soliton we follow a rotation of the local Dreibeins by $\pm 2 \pi$. This may answer a question, posed by Tsung-Dao Lee in a talk given in Vienna in the 1980s: "why does the mass break chiral symmetry?"

Besides its group theoretical properties, spin is a contribution to the total angular momentum. We will investigate these angular momentum properties in a dipole configuration. Since a dipole is uncharged, the field at infinity is independent of the direction, it approaches, for example, $\lim _{r \rightarrow \infty} Q(\mathbf{r})=-\mathrm{i} \sigma_{3}$. The symmetry of the vacuum is broken. We can combine the first configuration in Table 1 with the second to total spin $S=1$ or with the third to $S=0$. The energy for $S=0$ is slightly lower than that for $S=1$, the configuration shown in Figure 3. During a rotation of the dipole, the vacuum has to remain unchanged; a rigid rotation is not possible. If the dipole axis is rotated, for example, by

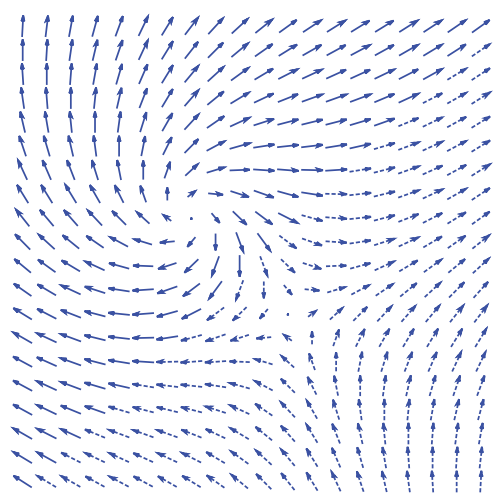

Figure 4: Configuration of Figure 3, after rotation by $\pi / 4$.

$\pi / 4$, as shown in Figure 4, the centers of the solitons have to rotate by the same angle. These rotations contribute to the total angular momentum.

Dipole configurations are not stable due to the attractive interaction between the charges. Their time evolution follows the general equations of motion [3]:

$$
\partial_{\mu}\left[\vec{\Gamma}_{\nu} \times \vec{R}^{\mu \nu}\right]+\vec{q} \frac{\mathrm{d} \Lambda}{\mathrm{d} q_{0}}=0
$$

derived from the Lagrangian (3). The energy-momentum tensor

$$
\Theta^{\mu}{ }_{v}=-\frac{\alpha_{f} \hbar c}{4 \pi}\left\{\left(\vec{\Gamma}_{v} \times \vec{\Gamma}_{\sigma}\right)\left(\vec{\Gamma}^{\mu} \times \vec{\Gamma}^{\sigma}\right)\right\}-\mathscr{L}(x) \delta_{v}^{\mu}
$$

turns out to be symmetric. There is no special symmetrisation necessary as in Maxwell's elecrodynamics [13]. Since there are no external forces, the force density is vanishing:

$$
f_{\nu}=\partial_{\mu} \Theta_{\nu}^{\mu}=0
$$

To determine a static potential one has to fix the positions of the centers at a chosen distance $d$. For distances $d \gg r_{0}$ the Coulombic behaviour is nicely reproduced, as can be seen in the diploma theses [14-16]. For distances $d \lesssim r_{0}$ the interaction strength increases. 


\section{Electrodynamic Limit}

One gets a pure Coulombic behaviour in the limit $r_{0} \rightarrow 0$ where one arrives at the Wu and Yang description [17] of dual Dirac monopoles by two degrees of freedom which we can choose as a normalised three-dimensional vector field $\vec{n}$. In this limit, we get

$$
\begin{gathered}
Q(x)=-\mathrm{i} \vec{\sigma} \vec{n}(x), \\
\vec{\Gamma}_{\mu}(x) \stackrel{(2)}{=} \vec{n}(x) \times \partial_{\mu} \vec{n}(x), \\
\vec{R}_{\mu \nu}(x) \stackrel{(3)}{=} \partial_{\mu} \vec{n}(x) \times \partial_{\nu} \vec{n}(x) .
\end{gathered}
$$

This is a description where the singularity of the Dirac string is removed, but the singularity of the Coulomb field is still present. The Lagrangian (3) reduces to (The same degrees of freedom but a different Lagrangian is used in the FadeevNiemi $=$ Skyrme-Fadeev $=$ Baby-Skyrme model $[18-20]$. The same Lagrangian was used in [21].)

$$
\mathscr{L}_{\mathrm{ED}}=-\frac{1}{4 \mu_{0}}{ }^{*} F_{\mu \nu}(x)^{*} F^{\mu \nu}(x)
$$

with the dual field strength tensor

$$
\begin{aligned}
{ }^{*} F_{\mu \nu}(x) & =-\frac{e_{0}}{4 \pi \varepsilon_{0} c} \vec{R} \\
\mu \nu & \vec{n} \\
& =-\frac{e_{0}}{4 \pi \varepsilon_{0} c} \vec{n}(x)\left[\partial_{\mu} \vec{n}(x) \times \partial_{\nu} \vec{n}(x)\right] .
\end{aligned}
$$

In this limit hedgehogs are characterised by point-like singularities in space and closed world-lines of line-like singularities in space-time:

$$
j^{\mu}=-e_{0} c \sum_{i=1}^{N} \int \mathrm{d} \tau_{i} \frac{\mathrm{d} X^{\mu}\left(\tau_{i}\right)}{\mathrm{d} \tau_{i}} \delta^{4}\left(x-X\left(\tau_{i}\right)\right)=(c \rho, \mathbf{j}) .
$$

Charges and fields are related by the inhomogeneous Maxwell equations:

$$
\frac{1}{2 \mu_{0}} \oint_{\partial V} \mathrm{~d} x^{\mu} \mathrm{d} x^{\nu *} F_{\mu \nu}=\frac{1}{6} \int_{V} \mathrm{~d} x^{\mu} \mathrm{d} x^{\nu} \mathrm{d} x^{\rho} \epsilon_{\mu \nu \rho \sigma} j^{\sigma} .
$$

Already here we see an essential difference to Maxwell's theory. Charges are quantized; there are no other charges possible than integer multiples of the elementary charge $e_{0}$. We get two further differences from the equations of motion:

$$
\partial_{\mu} \vec{n} g^{\mu}=0 .
$$

They allow for nonvanishing magnetic currents:

$$
g^{\mu}=c \partial_{\nu}{ }^{\star} f^{\nu \mu} \Longleftrightarrow\left\{\begin{array}{l}
\rho_{\mathrm{mag}}=\nabla \mathbf{B}, \\
\mathbf{g}=-\nabla \times \mathbf{E}-\partial_{t} \mathbf{B} .
\end{array}\right.
$$

The solutions of the homogeneous Maxwell equations fulfil the equations of motion (19). But there are further solutions possible which fulfil the relations:

$$
\begin{gathered}
\mathbf{B g} \stackrel{(19)}{=} 0, \\
c^{2} \mathbf{B} \rho_{\mathrm{mag}} \stackrel{(19)}{=} \mathbf{g} \times \mathbf{E},
\end{gathered}
$$

equivalent to the equations of motion (19). The presence of unquantized closed magnetic currents may be a discrepancy to experiments. But we could explain it with the observation, mentioned in the introduction, that in the experiment we only detect particles and never waves. We can speculate that such currents contribute to the recently intensively discussed dark matter. Further, we read from these equations that there are no solutions possible where $\mathbf{E}$ and $\mathbf{B}$ are parallel. This seems obviously also in contradiction to experiments, where it is rather simple to produce static parallel electric and magnetic fields. In this case it is more difficult to find an excuse. It could be that $\mathbf{E}$ and $\mathbf{B}$ are locally perpendicular and they appear to be parallel only in the average over space or time. This is the price to pay for restricting charges to integer multiples to the elementary charge and the fields to the two degrees of freedom of the $\vec{n}$-field.

\section{Coulomb and Lorentz Forces}

By the artificial splitting (16) of a single field $Q(x)$ in particles and their fields we reduce $\Theta^{\mu}{ }_{\nu}$ to the symmetric energymomentum tensor,

$$
T^{\mu}{ }_{\nu}(x) \stackrel{(12)}{=}-\frac{1}{\mu_{0}}{ }^{\star} F_{\nu \sigma}(x){ }^{\star} F^{\mu \sigma}(x)-\mathscr{L}_{\mathrm{ED}}(x) \delta_{\nu}^{\mu},
$$

and split the force density in two contributions

$$
f_{\nu}=\partial_{\mu} \Theta^{\mu}{ }_{\nu}=f_{\text {charges }}^{\mu}+\partial^{\nu} T^{\mu}{ }_{\nu} \stackrel{(13)}{=} 0,
$$

showing clearly the presence of Coulomb and Lorentz forces:

$$
\begin{aligned}
f_{\text {charges }}^{0} & =\frac{1}{c} \mathbf{j E}, \\
\mathbf{f}_{\text {charges }} & =\rho \mathbf{E}+\mathbf{j} \times \mathbf{B} .
\end{aligned}
$$

Here we would like to underline that the magnetic currents $\mathbf{g}$ do not contribute to electromagnetic forces.

\section{U(1) Gauge Invariance}

A U(1) gauge invariance appears as a rotational invariance by $\omega(x)$ around the $\vec{n}$-axis. By a rotation in colour space with

$$
\Omega(x)=\mathrm{e}^{\mathrm{i} \theta(x) \vec{e}_{\phi}(x) \vec{L}} \mathrm{e}^{\mathrm{i} \omega(x) \vec{n} \vec{L}} .
$$

We can rotate the $\vec{n}$-field in 3-direction:

$$
\vec{n}=\left(\begin{array}{c}
\sin \theta \cos \phi \\
\sin \theta \sin \phi \\
\cos \theta
\end{array}\right) \longrightarrow \vec{n}^{\prime}:=\Omega \vec{n}=\left(\begin{array}{l}
0 \\
0 \\
1
\end{array}\right)=\vec{e}_{3}
$$

Under this transformation the vector field $\vec{L} \vec{\Gamma}_{\mu}$ transforms to $\vec{L} \vec{\Gamma}_{\mu}^{\prime}=\Omega\left(\Gamma_{\mu}-\mathrm{i} \partial_{\mu}\right) \Omega^{\dagger}$ with

$$
\vec{\Gamma}_{\mu}^{\prime}=\left[(1-\cos \theta) \partial_{\mu} \phi+\partial_{\mu} \omega\right] \vec{e}_{3} .
$$


The curvature $\vec{R}_{\mu \nu}$ gets rotor form and turns out to be invariant against the rotations with $\omega(x)$ :

$$
\begin{aligned}
\vec{R}_{\mu \nu} & =\partial_{\mu} \vec{\Gamma}_{\nu}^{\prime}-\partial_{\nu} \vec{\Gamma}_{\mu}^{\prime} \\
& =\left[-\partial_{\mu} \cos \theta \partial_{\nu} \phi+\partial_{\nu} \cos \theta \partial_{\mu} \phi\right] \vec{e}_{3}
\end{aligned}
$$

The far field of a hedgehog soliton is the electric field strength of a classical electron and the vector field of a dual Dirac monopole:

$$
\begin{gathered}
\vec{E}_{r}^{\prime}=\frac{e_{0}}{4 \pi \varepsilon_{0}} \frac{\vec{e}_{3}}{r^{2}}, \\
\frac{\vec{\Gamma}_{\varphi}(\vartheta)}{r \sin \vartheta}=\frac{1-\cos \vartheta}{r \sin \vartheta} \vec{e}_{3}
\end{gathered}
$$

with a Dirac string along the 3 -direction.

\section{Hopf Number}

In Figure 2(a), we realised that the field lines of a point charge are lines of constant $\vec{n}$-field. We find also this relation for the dipole fields in Figures 3 and 4. Since in our model the vacuum has broken symmetry the field at infinity, " $\infty$ " is independent of the direction. Thus we get the isomorphism $\mathbb{R}^{3} \cup \infty \sim \mathcal{S}^{3}$. Due to the topological relation $\pi_{3}\left(\mathcal{S}^{2}\right)=\mathbb{Z}$ there is an additional quantum number for the $\vec{n}$-field, the Hopf number, or Gauß linking number $v$ of fibres $\mathscr{F}$ defined by $\vec{n}_{\mathscr{F}}=$ const and thus by certain values $\theta_{\mathscr{F}}$ and $\phi_{\mathscr{F}}$. This linking number is especially interesting in regions where we can neglect the influence of charges, in regions of pure $\vec{n}$ field, where the Lagrangian reduces to $\mathscr{L}_{\mathrm{ED}}$ of (16). According to the Hobart-Derrick theorem $[11,12]$ such configurations are unstable, if they move with a velocity slower than the speed of light $c$. Moving with $c$ they have an action constant in time and can be used to describe electromagnetic waves. For simplicity we choose the $z$-axis in the direction of motion; then it is sufficient to describe these configurations in $\mathbb{R}^{3}$ given by the coordinates $x, y$, and $\zeta=z-c t$. The linking number $v$ is a topological invariant. Its natural physical equivalent is the number of photons $n_{\gamma}$ in this configuration. As an example of such a configuration in $\mathbb{R}^{3}$ with $v=1$ we defined in the diploma thesis [22] a field by an area preserving map of $\mathcal{S}^{2}$ to a circle rotating with $\varphi$ around the line $\rho=\rho_{0}=$ $3, \zeta=0$, defined in cylindrical coordinates $\rho, \varphi, \zeta$ by

$$
\begin{aligned}
& \cos \theta= \begin{cases}\frac{\left(\rho-\rho_{0}\right)^{2}+\zeta^{2}}{2}-1 \leq 1 & \\
1 & \text { else }\end{cases} \\
& \phi=\varphi+\arctan \frac{\zeta}{\rho-\rho_{0}} .
\end{aligned}
$$

For a given $\vec{n}$-field one can get the linking number in $\mathbb{R}^{3}$ by the famous formula of Carl Friedrich Gauß:

$$
v=\frac{1}{4 \pi} \oint_{\mathscr{C}_{1}} \oint_{\mathscr{C}_{2}} \frac{\mathbf{r}_{1}-\mathbf{r}_{2}}{\left|\mathbf{r}_{1}-\mathbf{r}_{2}\right|^{3}} \cdot\left(\mathrm{d} \mathbf{r}_{1} \times \mathrm{d} \mathbf{r}_{2}\right),
$$
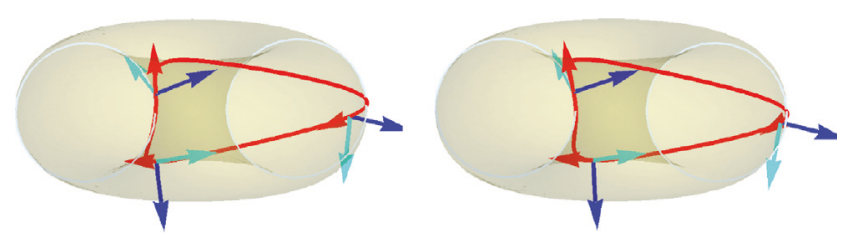

(a)
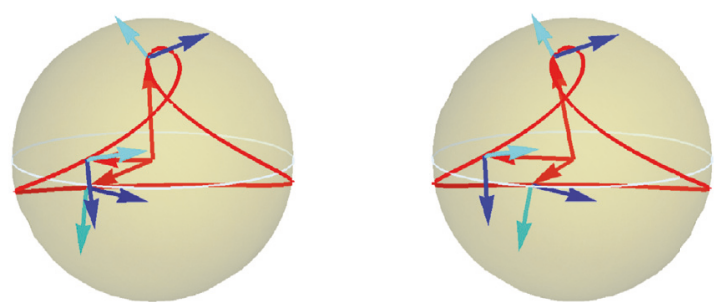

(b)

FIGURE 5: Stereographic view with parallel eyes at a fibre neighbourhood. (a) Fibre $\mathscr{F}$ on a torus in $\mathbb{R}^{3}$. Dreibeins of red tangential vectors and blue and cyan tangential vectors are drawn at three positions. (b) Gauß map $\mathscr{C}_{\mathscr{F}}$ of $\mathscr{F}$ with red radial vector $\vec{e}_{\mathscr{F}}^{\prime}(s)$ and blue and cyan tangential vectors at the corresponding three positions.

by a double integral. We get from the observation of the neighbourhood of a single fibre $\mathscr{F}$ another determination of $v$, by a single integral. We parametrize the position along the fibre with some parameter $s$. We determine the tangential vector $\mathbf{e}_{\mathscr{F}}(s)$ to the fibre and a unit vector to some neighbouring fibre, for example, defined by the perpendicular component $\mathbf{e}_{\theta}(s):=\left.\left(\nabla \cos \theta_{\mathscr{F}} /\left|\nabla \cos \theta_{\mathscr{F}}\right|\right)\right|_{\perp}$ of the gradient $\nabla \cos \theta_{\mathscr{F}}(s)$. We get a local Dreibein with $\mathbf{e}_{\perp}(s):=\mathbf{e}_{\mathscr{F}}(s) \times$ $\mathbf{e}_{\theta}(s)$. Then we determine the rotational velocity along the fibre by

$$
\frac{\mathrm{d} \omega}{\mathrm{d} s}:=\mathbf{e}_{\perp} \cdot \nabla_{s} \mathbf{e}_{\theta}=\mathbf{e}_{\mathscr{F}}\left(\mathbf{e}_{\theta} \times \nabla_{s} \mathbf{e}_{\theta}\right)
$$

It turns out that the integrated rotational angle $\omega$ depends on the radius $R=\sqrt{\left(\rho-\rho_{0}\right)^{2}+\zeta^{2}}$ of the torus surrounded by the fibre

$$
\omega(R):=\oint_{\mathscr{F}} \frac{\mathrm{d} \omega}{\mathrm{d} s} \mathrm{~d} s \neq 2 \pi n, \quad n \in \mathcal{N}
$$

and is not an integer. Its dependence on $R$ is depicted in Figure 6. This is understandable from the Gauß map $\mathbf{e}_{\mathscr{F}}(s) \mapsto$ $\mathbf{e}_{\mathscr{F}}^{\prime}(s)$ and $\mathbf{e}_{\theta}(s) \mapsto \mathbf{e}_{\theta}^{\prime}(s)$ to the $\delta_{\mathrm{G}}^{2}$ unit sphere; see Figure 5 . $\mathbf{e}_{\mathscr{F}}^{\prime}(s)$, plotted from the origin of $\mathcal{S}_{\mathrm{G}}^{2}$ by $\vec{e}_{\mathscr{F}}^{\prime}(s)=\vec{e}_{\mathscr{F}}(s)$, draws a curve $\mathscr{C}_{\mathscr{F}}$ on $\delta_{\mathrm{G}}^{2}$ (With bold symbols e we indicate the vectors and with $\vec{e}$ the set of its coordinates.). $\mathbf{e}_{\theta}^{\prime}(s)$ and $\mathbf{e}_{\perp}^{\prime}(s)$ are defined as vectors in the tangential space at $\mathbf{e}_{\mathscr{F}}(s)$ by the coordinate equalities $\vec{e}_{\theta}^{\prime}(s)=\vec{e}_{\theta}(s)$ and $\vec{e}_{\perp}^{\prime}(s)=$ $\vec{e}_{\perp}(s)$. Their parallel transport along $\mathscr{C}_{\mathscr{F}}$ is nontrivial, except for $\mathscr{C}_{\mathscr{F}}$ that is a great circle. Deviations from a great circle lead to an additional contribution of the curved geometry on $\mathcal{S}_{\mathrm{G}}^{2}$ to the rotational angle. Indicating the position on $\mathbb{S}_{\mathrm{G}}^{2}$ in 


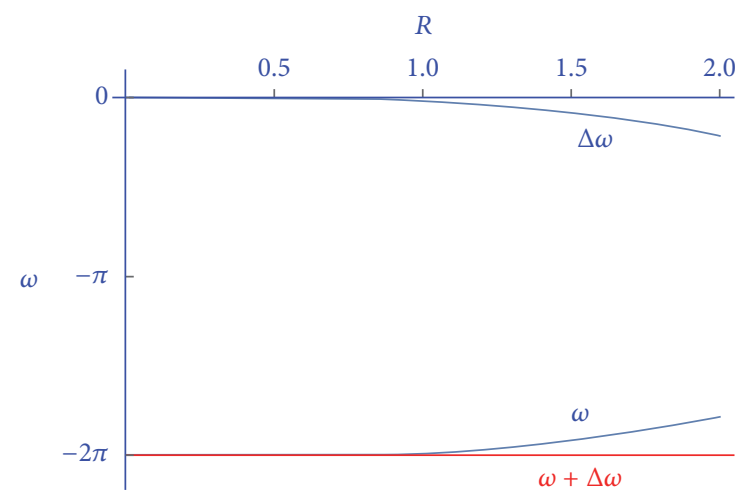

FIgURE 6: Contributions $\omega(R)$ and $\Delta \omega(R)$ to the determination of the rotational angle of the fibre neighbourhood.
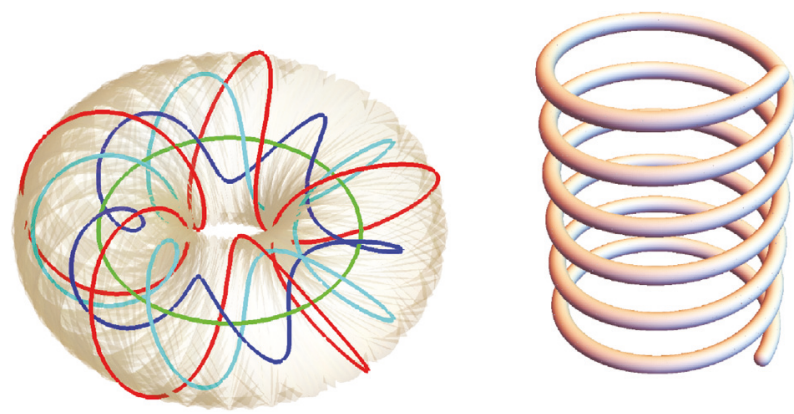

Figure 7: Configurations with higher linking numbers arranged with the shape of a torus or in spiral form.

spherical coordinates $\vec{e}_{\mathscr{F}}^{\prime}=(\sin \alpha \cos \beta, \sin \alpha \sin \beta, \cos \alpha)$, we can derive, for the corresponding spherical coordinate bases $\mathbf{e}_{\alpha}$ and $\mathbf{e}_{\beta}$, the affine $\mathrm{U}(1)$ connection:

$$
\begin{aligned}
& C_{\alpha}=0, \\
& C_{\beta}=\frac{\cos \alpha}{\sin \alpha}, \\
& \vec{C}=C_{\alpha} \vec{e}_{\alpha}+C_{\beta} \vec{e}_{\beta}
\end{aligned}
$$

which is trivial for meridians and the equator of $\mathcal{S}_{\mathrm{G}}^{2}$. The additional contribution reads

$$
\Delta \omega(R):=\oint \mathrm{d} s \frac{\mathrm{d} \vec{e}_{\mathscr{F}}^{\prime}}{\mathrm{d} s} \cdot \vec{C}
$$

The sum of both contributions gives then the expected value:

$$
\omega(R)+\Delta \omega(R)=2 \pi v .
$$

Both contributions of our example are shown in Figure 6; they give $v=-1$.

With the conjecture that the photon number of a configuration is given by the Gauß linking number $v$ of fibres, configurations with higher linking numbers $v$ correspond to states with several photons. In such configurations, see Figure 7; the linked fibres are spiraling several times. The

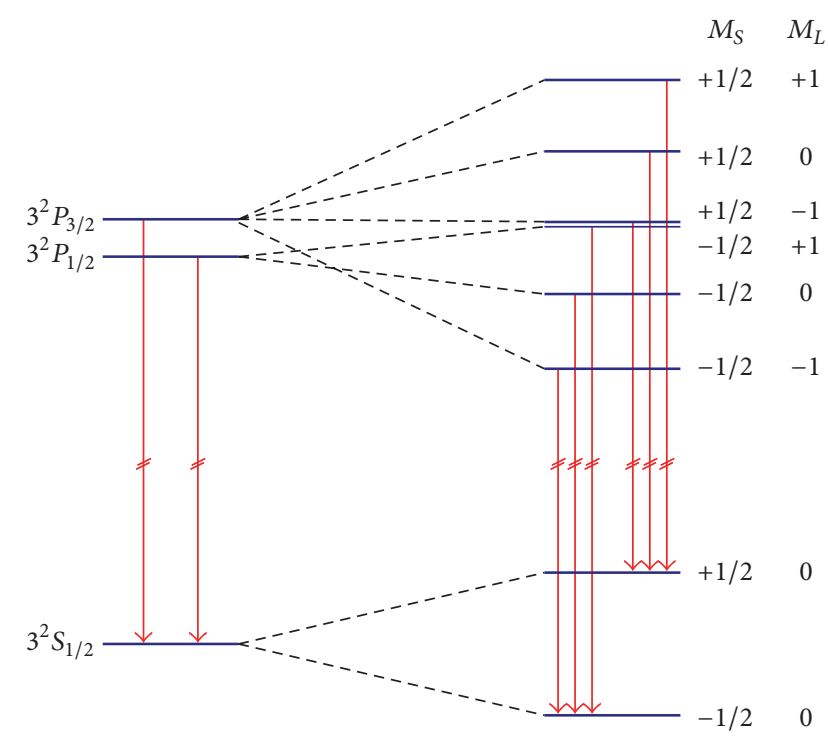

FIGURE 8: In a strong magnetic field the Na-D-line splits into three spectral lines with $\Delta M_{L}=0, \pm 1$ and $\Delta M_{S}=0$.
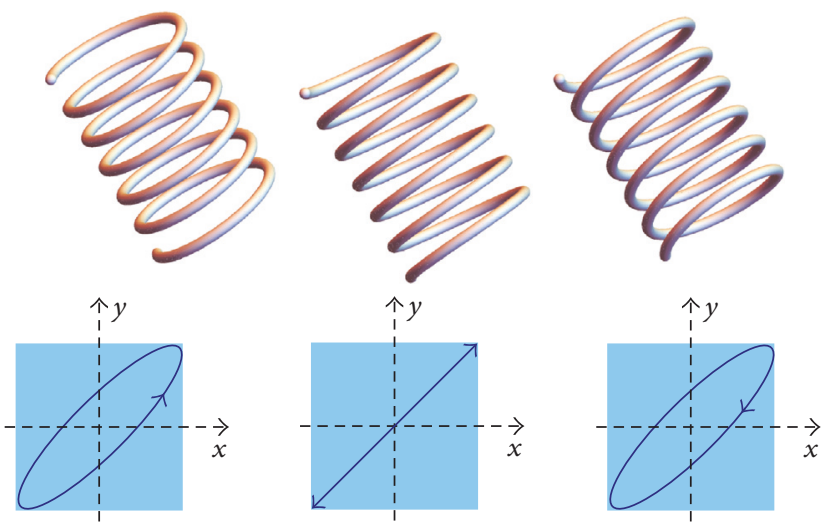

FIGURE 9: Different projections of rotations around a circle produce different polarisations.

spiral in the right diagram of Figure 7 reminds us of circular polarised waves.

It may be helpful to have a look at experiments. The NaD-line in a strong magnetic field splits into three lines, the Lorentz triple (see Figure 8), according to $\Delta M_{L}=0, \pm 1$. It is interesting to observe the three spectral lines parallel and perpendicular to the magnetic field, as depicted in Figure 10. The lines with $\Delta M_{L}=0$, the " $\pi$-components," are linearly polarised with an azimuth dependency of the intensity $I(\vartheta)=\sin ^{2} \vartheta$. The $\Delta M_{L}= \pm 1$-lines, the " $\sigma$ components," are circularly polarised with $I(9)=(1 / 2)(1+$ $\cos ^{2} 9$ ). If the circular polarised lines are observed in the direction perpendicular to the $\mathbf{B}$-field they appear linearly polarised. This reminds us of different projections of circular motion, of a spiral in the comoving $x, y, z-c t$ frame; see Figure 9.

Here a problem may appear. Rotations of a spiral do not change their chirality. After a rotation by $\pi$ a right-handed 


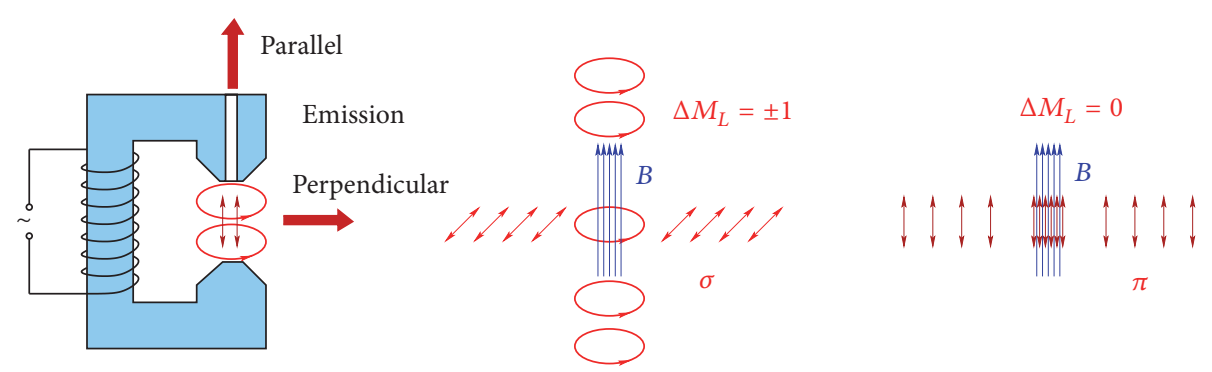

Figure 10: Scheme of observation of the Na-D-line in a strong magnetic field.

spiral remains right-handed as one can easily check with a right-handed thread of a screw. It is well-known that right polarised light can be easily transformed to linear polarised and to left polarised light by quarter-wave plates. These plates have perpendicular fast and slow axes.

This looks as a counterargument for the interpretation of the Hopf number as photon number. But it may also be an unknown feature of polarised light? Looking carefully at polarisation filters, one realises that filters exist for linear polarised light only! Right and left circular polarised light is detected by transforming it with quarter-wave plates, by interaction with matter, to linear polarisation.

\section{Conclusion}

The model described in this article is based on a field of $\mathrm{SO}(3)$-matrices in 3+1D Minkowski space-time. Therefore, the model has 3 degrees of freedom only, corresponding to the 3 Euler angles. These rotational matrices can be interpreted as describing the field of orientations of spatial Dreibeins at the points of space-time. In this sense, this simple model needs only the degrees of freedom of space and time to describe the various phenomena discussed in this article. To formulate the algebra of this model we are using the simpler representation of rotations by $\mathrm{SU}(2)$-matrices. As a consequence of this treatment we have to take into account the fact that for every $\mathrm{SO}(3)$-configuration there exist two SU(2)-configurations representing the same field. Vector fields (potentials) and tensor fields (field strength) are derived from the basic soliton field $Q(x)$.

The Lagrangian (3) of the model contains two terms. The kinetic term is proportional to the square of the curvature $\vec{R}_{\mu \nu}$ or to the dual field strength. Such a term is well-known from electrodynamics and QCD. The potential term reminds us of the Higgs potential and the cosmological constant. According to the Hobart-Derrick theorem a quarter of the mass of solitons would contribute with its average to the cosmological constant. The transition from $Q=1$ and $\Lambda=$ $1 / r_{0}^{4}$ to $\Lambda=0$ releases an energy density of $\alpha_{f} \hbar c /\left(4 \pi r_{0}^{4}\right)=$ $4.8 \mathrm{keV} / \mathrm{fm}^{3}=7.7 \cdot 10^{29} \mathrm{~J} / \mathrm{m}^{3}$ and could have contributed to inflation. Further, the potential term allows for a twodimensional degeneracy of the vacuum states.

In this model, there appear particle-like excitations as topological solitons. Their mass is field energy only. The field configurations are characterised by three topological quantum numbers which could find a physical realisation as electric charge, spin quantum number, and photon number. The topological structure does not allow two solitons to occupy the same space. This could be the topological origin of the Pauli principle. Charges appear as integer multiples of an elementary charge. No fractional charges are possible for stable excitations. Charges are characterised by regions where the spatial Dreibein rotates by $2 \pi$. Such regions interact via Coulomb and Lorentz forces. The electric force follows the $1 / r^{2}$ behaviour at large distances and increases for high momentum transfers. The distinction between charges and their fields is unnecessary. Such a distinction can be introduced as an approximation and simplifies the comparison with our physical experience. Eigenangular momentum (spin) appears as a consequence of orbital motion. The degenerate vacuum allows for two types of massless excitations, which could be related to the two polarisations of photons. $\mathrm{U}(1)$ gauge invariance emerges in the electrodynamic limit as rotational invariance of the above-mentioned Dreibein around the $\vec{n}$-axis.

Despite the small number of degrees of freedom there are further nonquantized disturbances possible. Magnetic currents as violations of the homogeneous Maxwell equations propagate with the speed of light and contribute to force fields only via their field strengths. Further we find waves in the rotational angle $\omega=2 \alpha$ with energy contributions from the potential term in the Hamiltonian. The cosmological discoveries of recent years allow attributing these two types of disturbances to dark energy and dark matter contributions.

The investigations seem to support the conjecture that the particles we find in experiments are topological solitons characterised by topological quantum numbers. They produce only spots on films and clicks in detectors. Waves seem to escape and are not directly detected. Waves could disturb the paths of particles and could contribute to a subquantum medium leading to quantum mechanics. In analogy to Couder's silicon oil drop experiments [23-26], the interference of waves created by the particle themselves with the vacuum waves could serve as a guiding wave field for particles.

If this model reproduced some features of nature, the two types of long-range forces which we find, gravitational and Coulomb forces, would be described in a geometrical manner with the degrees of freedom of space-time only. This would give a hint that particle physics could be closely related to gravitation. 


\section{Conflicts of Interest}

The author declares that there are no conflicts of interest regarding the publication of this paper.

\section{References}

[1] M. Remoissenet, Waves Called Solitons: Concepts and Experiments, Advanced Texts in Physics, Springer, Berlin, Germany, 2003.

[2] C. W. Misner, K. S. Thorne, and J. A. Wheeler, Gravitation, W. H. Freeman, San Francisco, Calif, USA, 1973, Number Teil 3 in Gravitation.

[3] M. Faber, "Model for topological fermions," Few-Body Systems, vol. 30, no. 3, pp. 149-186, 2001.

[4] M. Faber and A. P. Kobushkin, "Electrodynamic limit in a model for charged solitons," Physical Review D, vol. 69, no. 11, Article ID 116002, 2004.

[5] D. Borisyuk, M. Faber, and A. Kobushkin, "Electro-magnetic waves within a model for charged solitons," Journal of Physics A: Mathematical and Theoretical, vol. 40, no. 3, pp. 525-531, 2007.

[6] M. Faber, A. Kobushkin, and M. Pitschmann, "Shape vibrations of topological fermions," Advanced Studies in Theoretical Physics, vol. 2, pp. 11-22, 2008.

[7] M. Faber, "Particles as stable topological solitons," Journal of Physics: Conference Series, vol. 361, no. 1, Article ID 012022, 2012.

[8] M. Faber, "Spin and charge from space and time," Journal of Physics: Conference Series, vol. 504, no. 1, Article ID 012010, 2014.

[9] B. O. Rodrigues, "Des lois géométriques qui régissent les déplacements d'un système solide dans l'espace, et de la variation des coordonnées provenant de ces déplacements considérés indépendamment des causes qui peuvent les produire," Journal de Mathématiques Pures et Appliquées, vol. 5, pp. 380$440,1840$.

[10] T. H. R. Skyrme, "A non-linear field theory," Proceedings of the Royal Society A: Mathematical, Physical and Engineering Sciences, vol. 260, pp. 127-138, 1961.

[11] R. H. Hobart, "On the instability of a class of unitary field models," Proceedings of the Physical Society, vol. 82, no. 2, pp. 201-203, 1963.

[12] G. H. Derrick, "Comments on nonlinear wave equations as models for elementary particles," Journal of Mathematical Physics, vol. 5, pp. 1252-1254, 1964.

[13] J. D. Jackson, Classical Electrodynamics, Wiley, New York, NY, USA, 3rd edition, 1999.

[14] J. Wabnig, Interaction in the model of topological fermions [M.S. thesis], Technische Universität Wien, Wien, Austria, 2001.

[15] J. Resch, Numerische analyse an dipolkonfigurationen im modell topologischer fermionen [M.S. thesis], Technische Universität Wien, Wien, Austria, 2011.

[16] D. Theuerkauf, Charged particles in the model of topological fermions [M.S. thesis], Technische Universität Wien, Wien, Austria, 2016.

[17] T. T. Wu and C. N. Yang, "Some remarks about unquantized non-abelian gauge fields," Physical Review D, vol. 12, pp. 38433844, 1975.

[18] L. D. Faddeev, "Quantization of solitons," in Proceedings of the Tbilisi Conference, 1975.

[19] L. D. Faddeev, "Some comments on the many-dimensional solitons," Letters in Mathematical Physics, vol. 1, no. 4, pp. 289293, 1976.
[20] L. A. Ferreira and A. V. Razumov, "Hopf solitons and areapreserving diffeomorphisms of the sphere," Letters in Mathematical Physics, vol. 55, no. 2, pp. 143-148, 2001.

[21] L. A. Ferreira, "Exact time dependent Hopf solitons in $3+1$ dimensions," Journal of High Energy Physics, vol. 2006, 2006.

[22] J. Markus, Die hopfzahl in einer SU(2)-feldtheorie [M.S. thesis], Technische Universität Wien, Wien, Austria, 2014.

[23] Y. Couder, E. Fort, C.-H. Gautier, and A. Boudaoud, "From bouncing to floating: Noncoalescence of drops on a fluid bath," Physical Review Letters, vol. 94, no. 17, Article ID 177801, 2005.

[24] Y. Couder and E. Fort, "Single-particle diffraction and interference at a macroscopic scale," Physical Review Letters, vol. 97, no. 15, Article ID 154101, 2006.

[25] A. Eddi, E. Fort, F. Moisy, and Y. Couder, "Unpredictable tunneling of a classical wave-particle association," Physical Review Letters, vol. 102, no. 24, Article ID 240401, 2009.

[26] E. Fort, A. Eddi, A. Boudaoud, J. Moukhtar, and Y. Couder, "Path-memory induced quantization of classical orbits," Proceedings of the National Academy of Sciences of the United States of America, vol. 107, no. 41, pp. 17515-17520, 2010. 

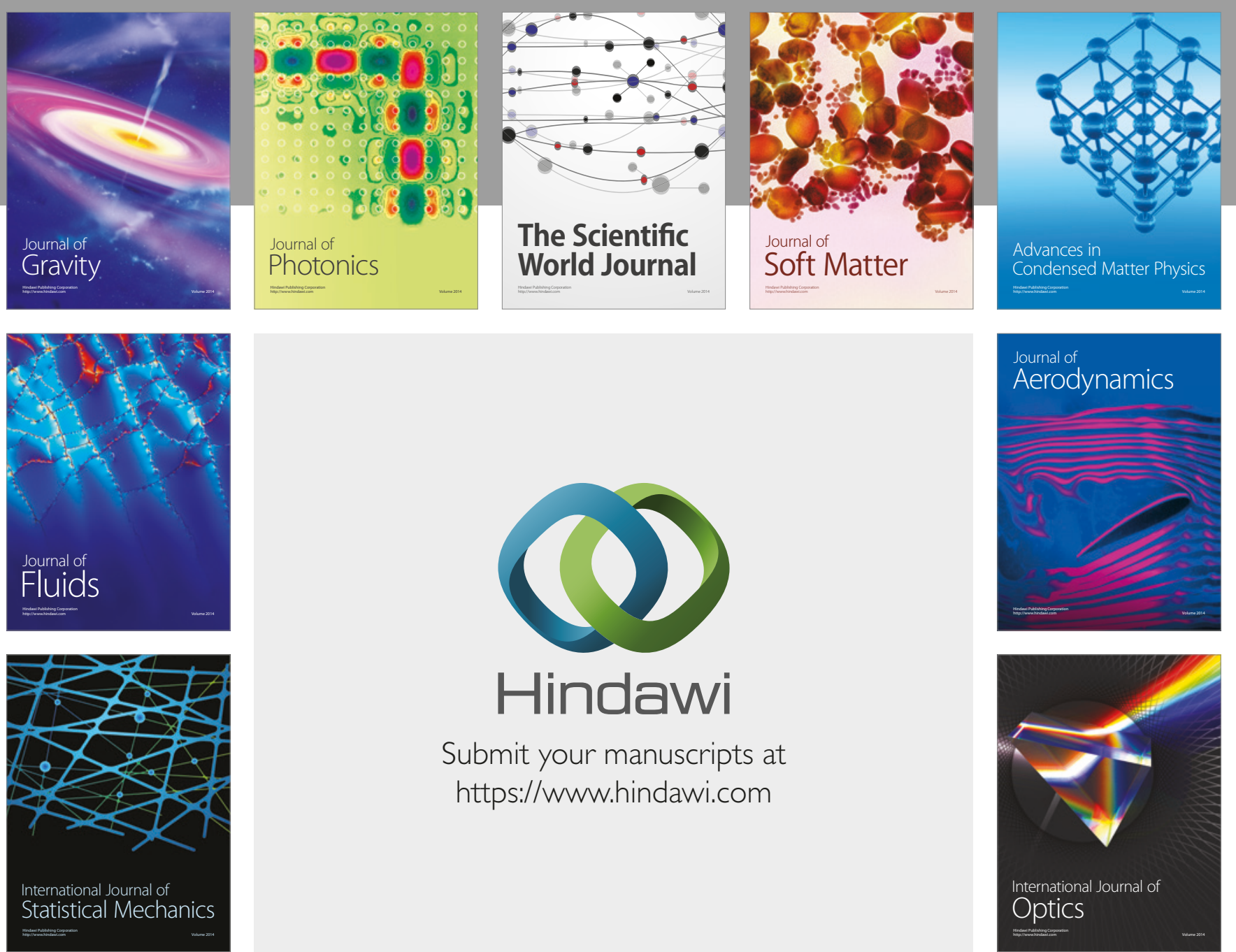

Submit your manuscripts at

https://www.hindawi.com
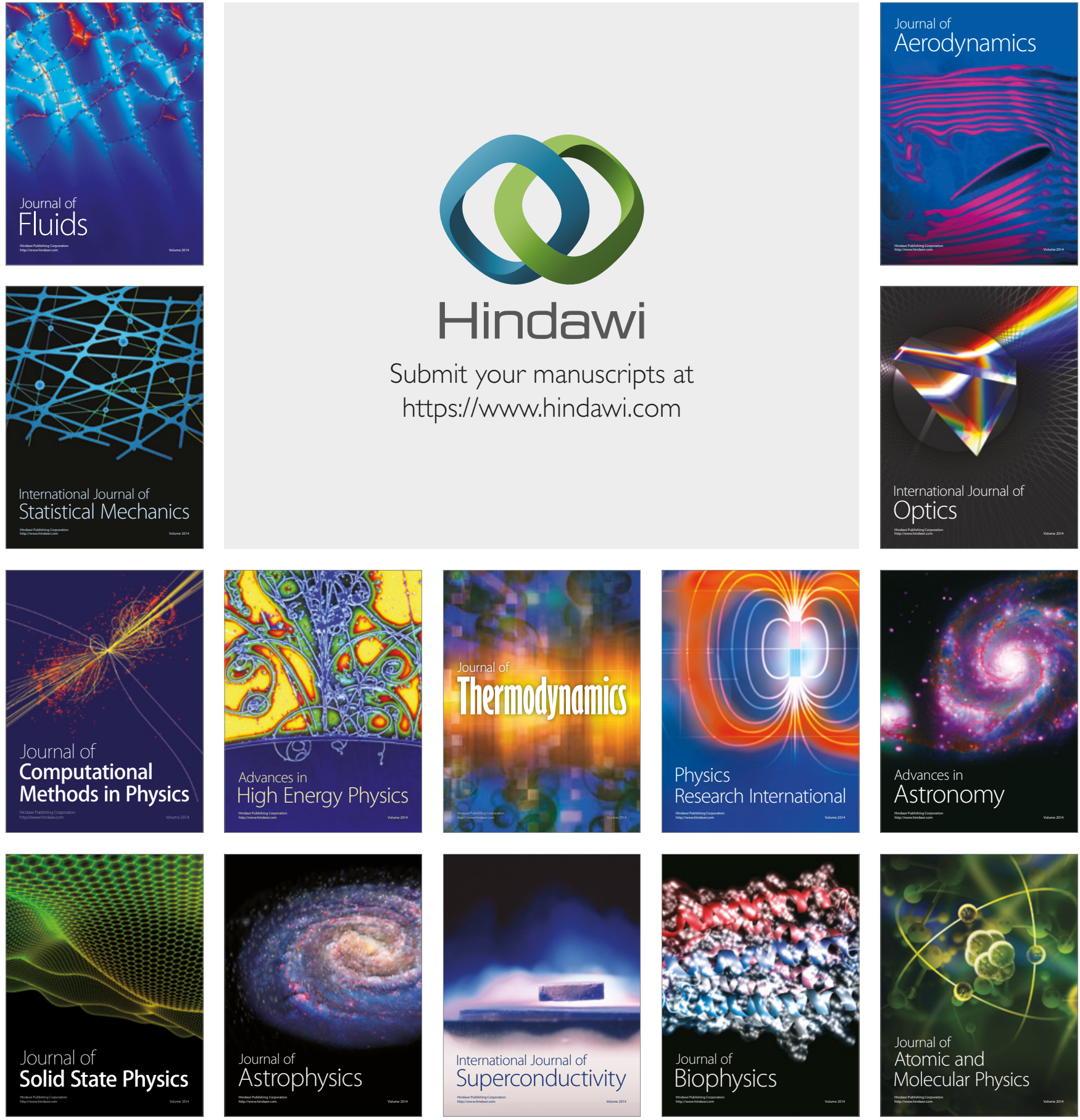